\title{
Solvothermal-Assisted Exfoliation Process to Produce Graphene with High Yield and High Quality
}

\author{
Wen Qian ${ }^{1, \dagger}$, Rui Hao ${ }^{1, \dagger}$, Yanglong Hou ${ }^{1}(\bowtie)$, Yuan Tian $^{2}$, Chengmin Shen ${ }^{2}$, Hongjun Gao ${ }^{2}$, and Xuelei Liang ${ }^{3}$ \\ ${ }^{1}$ Department of Advanced Materials and Nanotechnology, College of Engineering, Peking University, Beijing 100871, China \\ ${ }^{2}$ Institute of Physics, Chinese Academy of Sciences, Beijing 100190, China \\ ${ }^{3}$ Key Laboratory for the Physics and Chemistry of Nanodevices and Department of Electronics, Peking University, Beijing 100871, China \\ Received: 30 June 2009 / Revised: 24 July 2009 / Accepted: 24 July 2009 \\ CTsinghua University Press and Springer-Verlag 2009. This article is published with open access at Springerlink.com
}

\begin{abstract}
Monolayer and bilayer graphene sheets have been produced by a solvothermal-assisted exfoliation process in a highly polar organic solvent, acetonitrile, using expanded graphite (EG) as the starting material. It is proposed that the dipole-induced dipole interactions between graphene and acetonitrile facilitate the exfoliation and dispersion of graphene. The facile and effective solvothermal-assisted exfoliation process raises the low yield of graphene reported in previous syntheses to $10 \mathrm{wt} \%-12 \mathrm{wt} \%$. By means of centrifugation at $2000 \mathrm{rpm}$ for $90 \mathrm{~min}$, monolayer and bilayer graphene were separated effectively without the need to add a stabilizer or modifier. Electron diffraction and Raman spectroscopy indicate that the resulting graphene sheets are high quality products without any significant structural defects.
\end{abstract}

\section{KEYWORDS}

Graphene, solvothermal-assisted exfoliation, monolayer and bilayer, high quality

\section{Introduction}

Graphene, as an emerging two-dimensional (2-D) structure of free-standing carbon atoms packed into a dense honeycomb crystal structure, is being predicted to have numerous potential applications [1-8] because of its unusual electron transport properties and other distinctive characteristics. Various methods have been developed to produce graphene, including mechanical [1], physical [9-11], and chemical methods [12-18]. Chemical production of graphene is facile and low-cost and is one of the favoured methods for producing graphene. Currently, three major carbon sources are used as starting materials for chemical production of graphene: graphite oxide (GO) [12, 13], expanded graphite (EG) $[14,15,19]$, and sieved graphite powder (SGP) [16-18, 20]. Although chemical reduction of GO can produce graphene [21-24], the product contains significant numbers of oxygencontaining functional groups and irreversible lattice defects. Graphene sheets exfoliated from EG and SGP have fewer defects and are more conductive than chemically reduced graphene

Address correspondence to hou@pku.edu.cn

$\dagger$ These authors contributed equally to this work. 
oxide [25]. Recently, Dai et al. reported that graphene exfoliated from EG could be suspended in 1,2-dichloroethane solution by functionalization with poly(m-phenylenevinylene-co-2,5-dioctoxyp-phenylenevinylene) (PmPV) [15]. More recently, the solvothermal route was used to synthesize graphene sheets by using ethanol and sodium as reagents [26]. Our group has also demonstrated that aqueous dispersions of graphene can be prepared using 7,7,8,8-tetracyanoquinodimethane (TCNQ) anion as a stabilizer and EG as the starting material [19]. Coleman et al. have previously reported the liquid phase exfoliation of SGP to produce graphene, but the yield of monolayer graphene was only $\sim 1$ wt\% [16]. Therefore, to obtain high-yield and highquality graphene sheets in the free state without any modifiers and/or any stabilizers, which is a prerequisite to exploring graphene as successor to silicon in microelectronics and as competitor applied in other various areas, remains a great challenge.

Up to now, highly polar organic solvents, such as $\mathrm{N}, \mathrm{N}$-dimethylformamide (DMF), $\mathrm{N}$-methyl-2pyrrolidone (NMP), and dimethyl sulfoxide (DMSO), have been widely used to form dispersions of stable single-walled carbon nanotubes (SWNTs) [27] and graphene [16]. It is generally believed that the dipoleinduced dipole interaction is an important factor for dispersing SWNTs in highly polar organic solvents [27], so we proposed that similar effects should occur between graphene and polar solvents. Herein, we report that monolayer and bilayer graphene can be readily produced by solvothermal-assisted exfoliation and dispersion in a highly polar organic solvent, acetonitrile $(\mathrm{ACN})$, by using EG as the starting material. By means of centrifugation at $600 \mathrm{rpm}$, we obtained monolayer and bilayer graphene with a yield of $10 w t \%-12 w t \%$. Furthermore, by tuning the centrifugation velocity, we were able to separate monolayer from bilayer graphene. During the whole procedure, no stabilizer or modifier was introduced, and therefore graphene retains its unique intrinsic properties. The procedure described here offers an effective and simple method to produce graphene with the high yield and high quality needed for future applications.

\section{Experimental}

Exfoliation of graphene: EG was prepared according to the method of Dai et al. [14]. Commercial expandable graphite (average diameter of $300 \mu \mathrm{m}$, 99\% purity, Beijing Invention Biology Engineering \& New Material Co. Ltd., Beijing) was rapidly heated to $1000{ }^{\circ} \mathrm{C}$ and maintained for $60 \mathrm{~s}$ under an atmosphere of forming gas $\left(5 \% \mathrm{H}_{2}\right.$ and $\left.95 \% \mathrm{Ar}\right)$. The resulting EG ( $1 \mathrm{mg})$ was added to ACN (20 $\mathrm{mL}$ ), and the resulting mixture was transferred to a Teflon-lined autoclave $(25 \mathrm{~mL})$ and maintained at $180{ }^{\circ} \mathrm{C}$ for $12 \mathrm{~h}$, during which time the internal pressure reached 1.1 $\mathrm{MPa}$ [28]. The reaction products were sonicated for $60 \mathrm{~min}$, finally forming a black suspension. Afterwards, the mixture was divided into two parts and centrifuged using a KUBOTA 3700 centrifuge at $600 \mathrm{rpm}(900 \mathrm{~g})$ or $2000 \mathrm{rpm}(3000 \mathrm{~g})$ for $90 \mathrm{~min}$. The supernatant after centrifugation was comprised of single- and/or double-layer sheets, and kept for further characterization. The precipitated samples were relatively thick graphite flakes and incompletely exfoliated graphite. Calculations of the fractions of graphene and graphite were based on the mass measurement of dispersed graphene in the supernatant and precipitated graphite which was deposited onto filter paper by vacuum filtration, respectively. Before calculating the yield of graphene, we usually repeated the solvothermal process several tens of times in order to combine the samples. Therefore, the mass of EG used for calculation of the yield was at least $10 \mathrm{mg}$. The accuracy of the electronic balance used was $1 / 100,000$, which is sufficient for our measurements.

Structural characterization and study of properties: Atomic force microscopic (AFM) measurements were performed to characterize the sheet thickness and dimensions using a Nanoscope III MultiMode SPM (Digital Instruments) operated in tapping mode. The samples used for AFM measurement were prepared by depositing the corresponding dispersions on $1 \mathrm{~cm} \times 1 \mathrm{~cm}$ sections of fresh exfoliated highly oriented pyrolitic graphite (HOPG). The strong interactions between graphene and HOPG make the graphene adsorb strongly on

\section{Springer}


the surface of HOPG after evaporation of solvent. The morphology and crystal structure of graphene were investigated using a Philips Tecnai F30 FEGTEM operated with an accelerating voltage of 300 $\mathrm{kV}$. Raman spectra were recorded on a RENISHAW inVia Confocal Laser Micro-Raman spectrometer using an $\mathrm{Ar}^{+}$laser with wavelength $514.5 \mathrm{~nm}$ at room temperature. X-ray photoelectron spectroscopy (XPS) measurements were carried out using a PHI Quantera X-ray photoelectron spectrometer, which used $\mathrm{Al}(\mathrm{K} \alpha)$ radiation as a probe.

\section{Results and discussion}

Figure 1 schematically illustrates the solvothermalassisted exfoliation and dispersion process of graphene sheets in ACN. Firstly, the expandable graphite was heated at $1000{ }^{\circ} \mathrm{C}$ under an atmosphere of forming gas $\left(5 \% \mathrm{H}_{2}\right.$ and $\left.95 \% \mathrm{Ar}\right)$ to obtain the $\mathrm{EG}$, in which the d-spacing of graphene was greatly enlarged. Subsequently, the EG was mixed with ACN in a Teflon-lined autoclave and heated to 180 ${ }^{\circ} \mathrm{C}$ for $12 \mathrm{~h}$, introducing $\mathrm{ACN}$ molecules into the interlayers of EG by a solvothermal process. After reaction, EG precipitated at the bottom of ACN, suggesting that $\mathrm{ACN}$ has diffused into interlayers of EG, because EG would be floating above most solvents under usual conditions due to its loose structure. Upon sonication, monolayer and bilayer graphene sheets were peeled off from EG and stably dispersed in $\mathrm{ACN}$ to form a gray solution. Compared with NMP, DMF, and DMSO, ACN is an ideal solvent for a solvothermal process due to its low boiling point of $81.6^{\circ} \mathrm{C}$, which generates a reaction environment with high pressure which favors the insertion of ACN molecules into the interlayers of EG. ACN will also promote the dispersion of graphene because of its strong dipole moment of 3.87 D [28]. After insertion of $\mathrm{ACN}$, the dipole-induced dipole interactions between graphene and $\mathrm{ACN}$ facilitate the exfoliation and dispersion of graphene. No N1s signal was observed in the XPS spectrum (Fig. S-1 in the Electronic Supplementary Material (ESM)) of treated graphene samples. showing that the solvothermal process does not introduce nitrogen on the surface of graphene. The unique character of the solvothermal-assisted exfoliation process, which distinguishes it from other chemical methods, is that no stabilizer or modifier is involved, thus retaining the intrinsic structure and properties of graphene.

In the post-treatment process, the incompletely exfoliated graphite and thick graphite flakes were precipitated by centrifugation at $600 \mathrm{rpm}$ for $90 \mathrm{~min}$; the supernatant, which is composed of monolayer and bilayer graphene, formed a homogeneous gray solution. By measuring the weight of dispersed graphene in supernatant and precipitated graphite deposited on filter paper by vacuum filtration we can calculate the fraction of graphene/graphite obtained

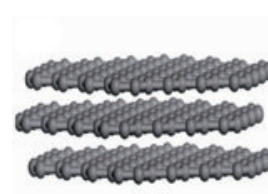

(a)

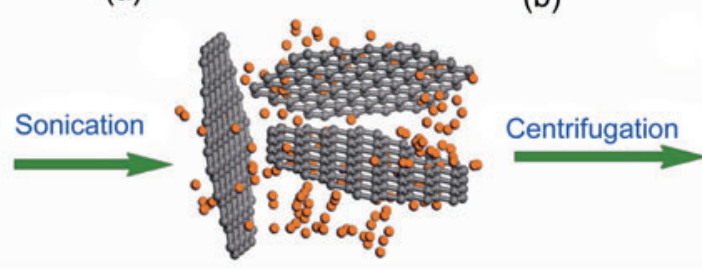

(d)

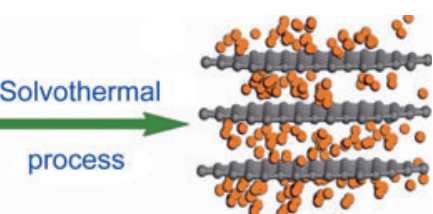

(c)

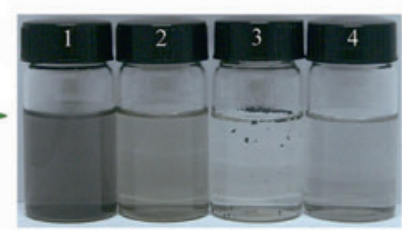

(e)
Figure 1 Schematic illustration of solvothermal-assisted exfoliation and dispersion of graphene sheets in ACN: (a) pristine expandable graphite; (b) EG; (c) insertion of ACN molecules into the interlayers of EG; (d) exfoliated graphene sheets dispersed in $A C N$; (e) optical images of four samples obtained under the different conditions described in Table 1

Table 1 Samples obtained by four different processes

\begin{tabular}{ccccc}
\hline No. & $\begin{array}{c}\text { Carbon } \\
\text { source }\end{array}$ & Process & $\begin{array}{c}\text { Centrifugation } \\
\text { conditions }\end{array}$ & Yield (wt\%) \\
\hline 1 & EG & Solvothermal-assisted process & $600 \mathrm{rpm}, 90 \mathrm{~min}$ & $10-12$ \\
2 & EG & Solvothermal-assisted process & $2000 \mathrm{rpm}, 90 \mathrm{~min}$ & $0.8-1$ \\
3 & EG & Without solvothermal-assisted process & $600 \mathrm{rpm}, 90 \mathrm{~min}$ & None \\
4 & SGP & Without solvothermal-assisted process & $600 \mathrm{rpm}, 90 \mathrm{~min}$ & $0.5-0.6$ \\
\hline
\end{tabular}


with different procedures, as listed in Table 1. In case of graphene sheets exfoliated from EG combined with a solvothermal process, the yield of graphene after centrifugation at $600 \mathrm{rpm}$ for $90 \mathrm{~min}$ was $10 \mathrm{wt} \%-12$ $w t \%$ (Sample 1). When the centrifugation was carried out at $2000 \mathrm{rpm}$ for $90 \mathrm{~min}$, with the same procedure, the yield of graphene was lower (Sample 2). For comparison, when graphene sheets were exfoliated from EG or SGP without an initial solvothermal process, no graphene was obtained in the case of EG (Sample 3) and the yield of graphene from SGP was also very low when the centrifugation was carried out at $600 \mathrm{rpm}$ for $90 \mathrm{~min}$ (Sample 4). The experimental results prove that it is difficult for ACN molecules to effectively insert into the interlayer spaces of graphene without a solvothermal process. In other words, by means of the solvothermal process, ACN molecules were supplied with sufficient energy to overcome the potential barrier, making them diffuse into the interlayers of EG more effectively, which is crucial to the exfoliation of graphene from EG.

AFM was employed to characterize the thickness and dimensional size of the exfoliated graphene sheets. In order to eliminate the possibility of any effects due to the HOPG substrate, we obtained an AFM image (Fig. S-2 in the ESM) of fresh exfoliated HOPG itself which confirmed that it was clean and flat. When the graphene sheets were centrifuged at $600 \mathrm{rpm}$ for $90 \mathrm{~min}$, the topographic height of the resulting graphene was between $0.5-1.2 \mathrm{~nm}$. Considering the intrinsic monolayer ripples owing to thermal fluctuations [29], it can be concluded that the graphene sheets centrifuged at $600 \mathrm{rpm}$ are a mixture of monolayers and bilayers, as shown in Figs. 2(a) and 2(b). After centrifugation at $2000 \mathrm{rpm}$ for $90 \mathrm{~min}$, the height profiles taken along the white lines in Figs. 2(c) and 2(d) showed the thickness of the graphene sheets was about $0.5-0.6 \mathrm{~nm}$, indicating that most of them are flat and monolayer. The lateral dimension of graphene ranges from 100 to $800 \mathrm{~nm}$. Transmission electron microscopy (TEM) and electron diffraction (ED) were also employed to investigate the morphology and crystal structure of the graphene samples. Figure 3(a) is a TEM image of monolayer graphene; the corresponding ED pattern exhibits a typical sixfold symmetry diffraction
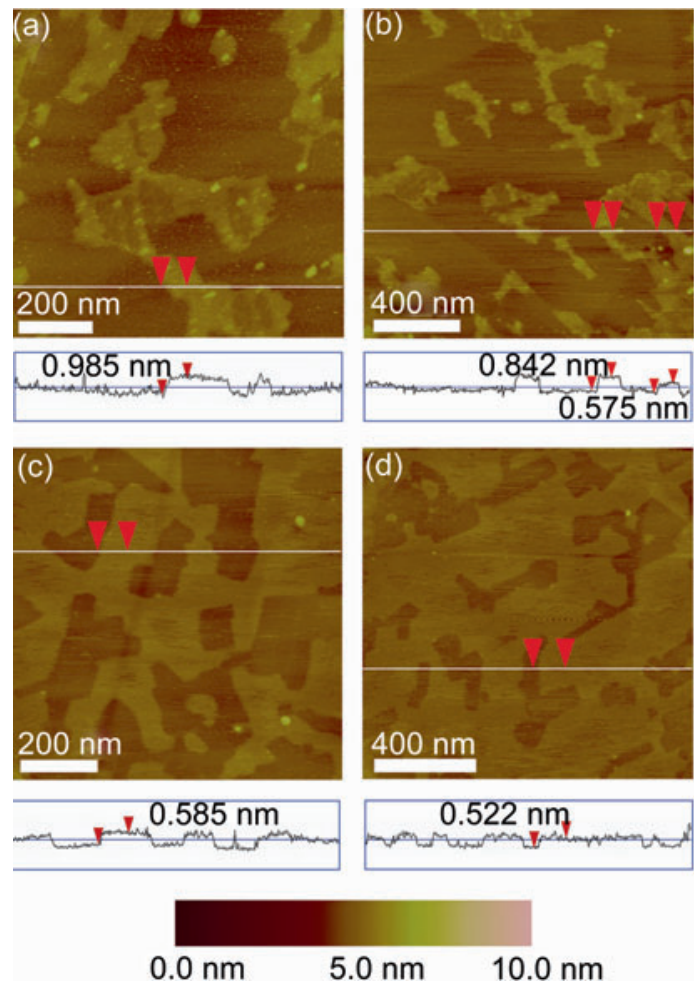

Figure 2 AFM height images of graphene in tapping mode (Height scale bar, 0-10 nm): (a), (b) monolayer and bilayer graphene sheets obtained by centrifugation at $600 \mathrm{rpm}$ for $90 \mathrm{~min}$, with a thickness of about 0.5-1.2 nm; (c), (d) monolayer graphene sheets obtained by centrifugation at $2000 \mathrm{rpm}$ for $90 \mathrm{~min}$, with a thickness of about $0.5-0.6 \mathrm{~nm}$
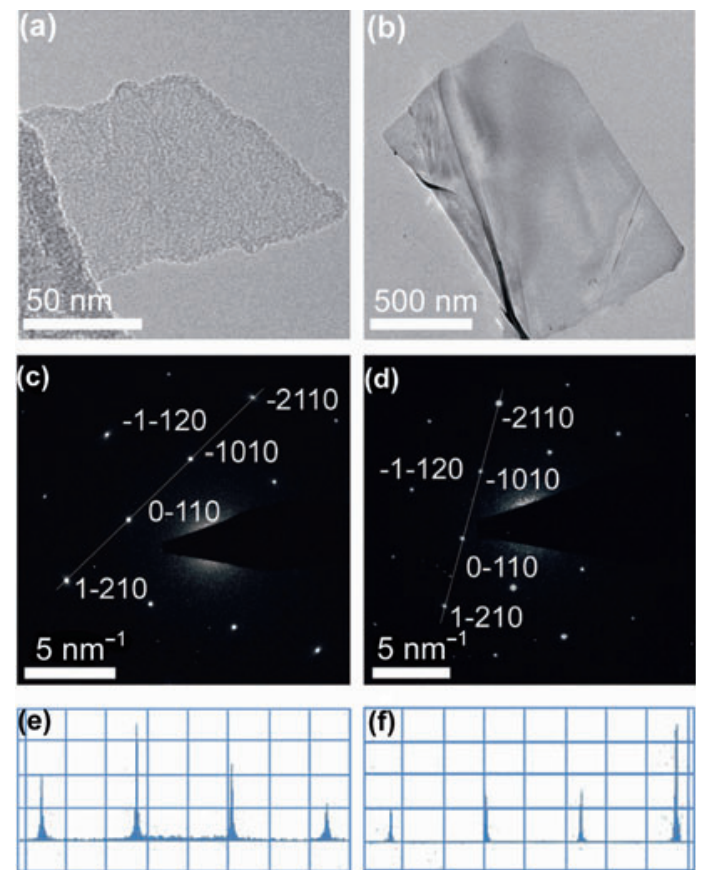

Figure 3 (a), (c) TEM image of monolayer graphene and the corresponding ED pattern; (b), (d) TEM image of bilayer graphene and the corresponding ED pattern; (e), (f) Intensity profile plots along the line indicated in (c) and (d), respectively 
pattern (Fig. 3(c)). Figure 3(b) shows a TEM image of bilayer graphene with edges tending to scroll and fold slightly, and the corresponding ED pattern is displayed in Fig. 3(d). The intensity profile of a line through the (1-210)-(0-110)-(-1010)-(-2110) axis for the pattern in Fig. 3(c) is plotted in Fig. 3(e). The inner peaks $\{1100\}$ are relatively more intense than the outer ones $\{2110\}$, with $I_{\{1100\}} / I_{\{2110\}}$ greater than 1 $(\sim 1.5)$, confirming that the graphene is monolayer. Conversely, Fig. 3(f) shows that the inner peaks $\{1100\}$ are less intense than the outer ones $\{2110\}$, giving a ratio of $I_{\{1100\}} / I_{\{2110\}}$ of less than $1(\sim 0.5)$, which is ascribed to bilayer graphene with predominantly $\mathrm{AB}$ stacking. This observation is consistent with previous computational results for graphene layers [30].

Raman spectroscopy has been accepted to be a very versatile, purely optical, high-throughput technique for the characterization of graphene. Through the analysis of the intensity, frequency, and line width of characteristic Raman modes, we are not only able to clearly distinguish singleand double-layer graphene from multi-layered (>5 layers) graphene films [31], but are also able to obtain valuable material properties of graphene, such as doping level and defect density. The Raman spectrum of graphene is dominated by three main features, G-, D-, and 2-D- Raman modes, each having different physical origins. The peak at $\sim 1580 \mathrm{~cm}^{-1}$ (G band), arising from emission of zone-centre optical phonons, corresponds to the doubly degenerate $E_{2 g}$ mode of graphite related to the vibration of $\mathrm{sp}^{2}$ bonded carbon atoms. The disorder-induced D (1350 $\mathrm{cm}^{-1}$ ) band and its symmetry-allowed 2-D overtone band $\left(2700 \mathrm{~cm}^{-1}\right)$ involve preferential coupling to transverse zone-boundary optical phonons. Figure 4 illustrates Raman spectra of precipitated graphite, and bilayer and monolayer graphene sheets. The evolution of the Raman spectra of graphene with the number of layers, especially the 2-D band, is very similar to that observed by Ferrari et al. [31]. The spectrum (Fig. 4(a)) of precipitated graphite exhibits two peaks at $1580 \mathrm{~cm}^{-1}$ and $2726 \mathrm{~cm}^{-1}$, which can be ascribed to thick flakes ( $>10$ layers). For graphene sheets after centrifugation at $600 \mathrm{rpm}$, the spectrum (Fig. 4(b)) exhibits two peaks at $1575 \mathrm{~cm}^{-1}$ and 2716 $\mathrm{cm}^{-1}$, i.e., the wavenumber of the $\mathrm{G}$ band is $5 \mathrm{~cm}^{-1}$ lower than that of the thick flakes and the 2-D band is $10 \mathrm{~cm}^{-1}$ lower. We use up to four components to fit the line shape of the 2-D band in the spectrum (Fig. $4(\mathbf{e}))$ which is in good agreement with the results for bilayer graphene [31]. In the case of graphene sheets after the centrifugation at $2000 \mathrm{rpm}$, the spectrum (Fig. 4(c)) shows a lower intensity of the G band $\left(1570 \mathrm{~cm}^{-1}\right)$, and a broader 2-D band compared with the corresponding peaks in the previous spectra (Figs. 4(a) and 4(b)). Different from Ferrari's results [31], where micro-Raman spectroscopy was used to measure a single graphene sheet, our Raman signal is an ensemble average of several graphene sheets. Thus, it is reasonable that the 2-D peak of graphene sheets after centrifugation at $2000 \mathrm{rpm}$ is broader than that of monolayer graphene due to inhomogeneous broadening (Fig. 4(f)). Furthermore, it is worth noting that all of these Raman spectra have small or invisible $\mathrm{D}$ bands, indicating that the solvothermal process does not introduce significant structural defects. Finally, we also measured the sheet resistance of graphene film by a four-probe instrument, and the resistance was about $1000 \Omega$ /sq (see ESM).

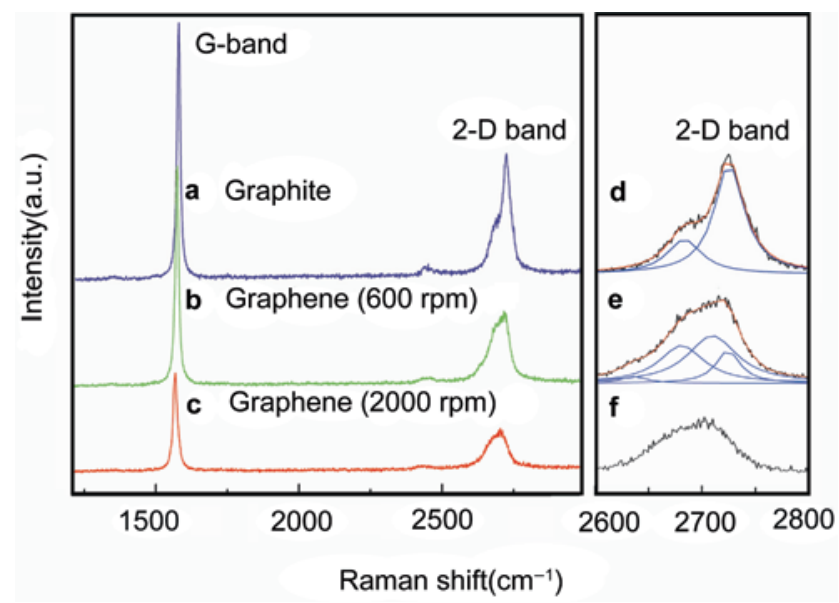

Figure 4 Raman spectra of (a) thick graphite flakes, (b) bilayer graphene and (c) monolayer graphene; (d)-(f) the enlarged spectra at the $2-D$ band of $(\mathbf{a})-(\mathbf{c})$, respectively

\section{Conclusions}

We report a simple route to produce graphene by a solvothermal-assisted exfoliation process in the presence of $\mathrm{ACN}$. Compared with previous procedures, this method results in greatly improved yields of monolayer and bilayer graphene sheets 
up to $10 \mathrm{wt} \%-12 \mathrm{wt} \%$. The defect-free nature of the resulting graphene samples was confirmed by ED and Raman spectroscopy. This solvothermal-assisted exfoliation process opens up an effective route to prepare high yields of high-quality graphene, which should be helpful in the development of various potential applications ranging from graphenebased composites, sensors, and biomedicines to nanodevices.

\section{Acknowledgements}

This work was partially supported by Beijing New Star Project of Science and Technology (2008B02), the Scientific Research Foundation for Returned Scholars from the Ministry of Education of China, the National Basic Research Program (2010CB934600) of China, Ministry of Science and Technology China and Startup Fund of Distinguished Young Scholars at Peking University. Dr. W Qian acknowledges the postdoctoral fellowship supported by the National Nature Science Foundation of China. We thank Prof. D. P Yu and Mr. Y. B. Zhou for their kind help.

Electronic Supplementary Material: Supplementary material is available in the online version of this article at http://dx.doi.org/10.1007/s12274-009-9074-z and is accessible free of charge.

\section{References}

[1] Novoselov, K. S.; Geim, A. K.; Morozov, S. V.; Jiang, D.; Zhang, Y.; Dubonos, S. V.; Grigorieva, I. V.; Firsov, A. A. Electric field effect in atomically thin carbon films. Science 2004, 306, 666-669.

[2] Lee, C.; Wei, X.; Kysar, J. W.; Hone, J. Measurement of the elastic properties and intrinsic strength of monolayer graphene. Science 2008, 321, 385-388.

[3] Di, C. A.; Wei, D. C.; Yu, G.; Liu, Y. Q.; Guo, Y. L.; Zhu, D. B. Patterned graphene as source/drain electrodes for bottom-contact organic field-effect transistors. Adv. Mater. 2008, 20, 3289-3293.

[4] Liu, Z. F.; Liu, Q.; Huang, Y.; Ma, Y. F.; Yin, S. G.; Zhang, X. Y.; Sun, W.; Chen, Y. S. Organic photovoltaic devices based on a novel acceptor material: Graphene. Adv. Mater. 2008, 20, 3924-3930.
[5] Ang, P. K.; Chen, W.; Wee, A. T. S.; Loh, K. P. Solutiongated epitaxial graphene as $\mathrm{pH}$ sensor. J. Am. Chem. Soc. 2008, 130, 14392-14393.

[6] Liu, Z.; Robinson, J. T.; Sun, X. M.; Dai, H. J. PEGylated nanographene oxide for delivery of water-insoluble cancer drugs. J. Am. Chem. Soc. 2008, 130, 1087610877.

[7] Chen, H.; Muller, M. B.; Gilmore, K. J.; Wallace, G. G.; $\mathrm{Li}, \mathrm{D}$. Mechanically strong, electrically conductive, and biocompatible graphene paper. Adv. Mater. 2008, 20, 3557-3561.

[8] Stankovich, S.; Dikin, D. A.; Dommett, G. H. B.; Kohlhaas, K. M.; Zimney, E. J.; Stach, E. A.; Piner, R. D.; Nguyen, S. T.; Ruoff, R. S. Graphene-based composite materials. Nature 2006, 442, 282-286.

[9] Berger, C.; Song, Z. M.; Li, X. B.; Wu, X. S.; Brown, N.; Naud, C.; Mayou, D.; Li, T. B.; Hass, J.; Marchenkov, A. N.; Conrad, E. H.; First, P. N.; de Heer, W. A. Electronic confinement and coherence in patterned epitaxial graphene. Science 2006, 312, 1191-1196.

[10] Pan, Y.; Zhang, H. G.; Shi, D. X.; Sun, J. T.; Du, S. X.; Liu, F.; Gao, H. J. Highly ordered, millimeter-scale, continuous, single-crystalline graphene monolayer formed on Ru (0001). Adv. Mater. 2009, 21, 2777-2780.

[11] Kim, K. S.; Zhao, Y.; Jang, H.; Lee, S. Y.; Kim, J. M.; Ahn, J. H.; Kim, P.; Choi, J. Y.; Hong, B. H. Large-scale pattern growth of graphene films for stretchable transparent electrodes. Nature 2009, 457, 706-710.

[12] Si, Y.; Samulski, E. T. Synthesis of water soluble graphene. Nano Lett. 2008, 8, 1679-1682.

[13] Xu, Y. X.; Bai, H.; Lu, G. W.; Li, C.; Shi, G. Q. Flexible graphene films via the filtration of water-soluble noncovalent functionalized graphene sheets. J. Am. Chem. Soc. 2008, 130, 5856-5857.

[14] Li, X. L.; Wang, X. R.; Zhang, L.; Lee, S. W.; Dai, H. J. Chemically derived, ultrasmooth graphene nanoribbon semiconductors. Science 2008, 319, 1229-1232.

[15] Li, X. L.; Zhang, G. Y.; Bai, X. D.; Sun, X. M.; Wang, X. R.; Wang, E.; Dai, H. J. Highly conducting graphene sheets and Langmuir-Blodgett films. Nat. Nanotechnol. 2008, 3 , 538-542.

[16] Hernandez, Y.; Nicolosi, V.; Lotya, M.; Blighe, F. M.; Sun, Z. Y.; De, S.; McGovern, I. T.; Holland, B.; Byrne, M.; Gun'ko, Y. K.; Boland, J. J.; Niraj, P.; Duesberg, G.; Krishnamurthy, S.; Goodhue, R.; Hutchison, J.; Scardaci, V.; Ferrari, A. C.; Coleman, J. N. High-yield production 
of graphene by liquid-phase exfoliation of graphite. Nat. Nanotechnol. 2008, 3, 563-568.

[17] Valles, C.; Drummond, C.; Saadaoui, H.; Furtado, C. A.; He, M.; Roubeau, O.; Ortolani, L.; Monthioux, M.; Penicaud, A. Solutions of negatively charged graphene sheets and ribbons. J. Am. Chem. Soc. 2008, 130, 15802 -15804 .

[18] Liu, N.; Luo, F.; Wu, H. X.; Liu, Y. H.; Zhang, C.; Chen, J. One-step ionic-liquid-assisted electrochemical synthesis of ionic-liquid-functionalized graphene sheets directly from graphite. Adv. Funct. Mater. 2008, 18, 1518-1525.

[19] Hao, R.; Qian, W.; Zhang, L. H.; Hou, Y. L. Aqueous dispersions of TCNQ-anion-stabilized graphene sheets. Chem. Commun. 2008, 6576-6578.

[20] Viculis, L. M.; Mack, J. J.; Kaner, R. B. A chemical route to carbon nanoscrolls. Science 2003, 299, 1361-1361.

[21] Fan, X. B.; Peng, W. C.; Li, Y.; Li, X. Y.; Wang, S. L.; Zhang, G. L.; Zhang, F. B. Deoxygenation of exfoliated graphite oxide under alkaline conditions: A green route to graphene preparation. Adv. Mater. 2008, 20, 44904493.

[22] Tung, V. C.; Allen, M. J.; Yang, Y.; Kaner, R. B. Highthroughput solution processing of large-scale graphene. Nat. Nanotechnol. 2009, 4, 25-29.

[23] Park, S.; An, J. H.; Piner, R. D.; Jung, I.; Yang, D. X.; Velamakanni, A.; Nguyen, S. T.; Ruoff, R. S. Aqueous suspension and characterization of chemically modified graphene sheets. Chem. Mat. 2008, 20, 6592-6594.

[24] Stankovich, S.; Piner, R. D.; Chen, X. Q.; Wu, N. Q.;
Nguyen, S. T.; Ruoff, R. S. Stable aqueous dispersions of graphitic nanoplatelets via the reduction of exfoliated graphite oxide in the presence of poly(sodium 4-styrenesulfonate). J. Mater. Chem. 2006, 16, 155-158.

[25] Zhu, J. Graphene production: New solutions to a new problem. Nat. Nanotechnol. 2008, 3, 528-529.

[26] Choucair, M.; Thordarson, P.; Stride, J. A. Gram-scale production of graphene based on solvothermal synthesis and sonication. Nat. Nanotechnol. 2009, 4, 30-33.

[27] Landi, B. J.; Ruf, H. J.; Worman, J. J.; Raffaelle, R. P. Effects of alkyl amide solvents on the dispersion of single-wall carbon nanotubes. J. Phys. Chem. B 2004, 108, 17089-17095.

[28] Please confirm. "World Publishing Corporation" should be revised as "Beijing World Publishing Corporation/ McGraw-Hill"

[29] Fasolino, A.; Los, J. H.; Katsnelson, M. I. Intrinsic ripples in graphene. Nat. Mater. 2007, 6, 858-861.

[30] Horiuchi, S.; Gotou, T.; Fuijwara, M.; Sotoaka, R.; Hirata, M.; Kimoto, K.; Asaka, T.; Yokosawa, T.; Matsui, Y.; Watanabe, K.; Sekita, M. Carbon nanofilm with a new structure and property. Jpn. J. Appl. Phys. Part 2. 2003, 42, (9AB), L1073-L1076.

[31] Ferrari, A. C.; Meyer, J. C.; Scardaci, V.; Casiraghi, C.; Lazzeri, M.; Mauri, F.; Piscanec, S.; Jiang, D.; Novoselov, K. S.; Roth, S.; Geim, A. K. Raman spectrum of graphene and graphene layers. Phys. Rev. Lett. 2006, 97, 187401187404. 